\title{
$\&$ Telemedicine for elective neurosurgical routine follow-up care: a promising patient-centered and cost-effective alternative to in-person clinic visits
}

\author{
Susan R. Snyder, PhD, MBA \\ Geisinger Department of Epidemiology and Health Services Research, Danville, Pennsylvania
}

$\mathrm{T}$ ELEmedicine, remote care of patients aided by internet- or telephone-based telecommunication technology, has been developed to overcome barriers to providing services, especially for patients needing timesensitive specialty care in rural or medical-shortage areas requiring long-distance travel.

\section{Telemedicine Benefits in Routine Postsurgical Follow-Up Care}

Telemedicine as used in postoperative follow-up care commonly relies on nonsurgeon medical and/or allied health professionals (e.g., nurse practitioners). When used for routine postsurgical follow-up care, telemedicine strategies offer the potential to narrow access to care and cost disparities during a critical time period when most complications occur, and telemedicine may also reduce gaps and frustrations that can negatively impact a patient's clinical outcomes and quality of life.?

Employing available telemedicine technologies is a strategy that can bridge care gaps and improve access to needed services for patients who can be cared for without their having to return to a regional specialty center. Local clinics, patient homes, and facilities with appropriate telemedicine technology can be used during the postoperative period for 3 general purposes: for follow-up visits that replace those scheduled at distant sites, ongoing monitoring, and the remote identification of complications. ${ }^{10}$ Relatively brief, structured telemedicine visits for some post-neurosurgical follow-up care can reduce the time and travel costs to patients and their families, and they can reduce stress. $^{8}$

Systematic review evidence of telemedicine intervention outcomes for postsurgical follow-up care comes from 21 mostly small US studies subject to selection bias in diverse low-risk surgery patients published between 2010 and July 2015. ${ }^{3}$ For the included studies that reported the following outcomes, the majority of patients were willing to use telemedicine, and there was high patient and provider satisfaction, and universal and significant savings in round-trip travel distance, time, and costs. Potential health care system benefits were also noted based on a single telephone follow-up study within the VA system ${ }^{4}$ in terms of cost savings, freed clinic time for new patients, and reduced wait times in specialty care with long surgery wait times. Although this evidence is not based on representative patient populations, many of the benefits of telemedicine for postsurgical patients treated by other specialties may also apply to neurosurgery.

\section{Telemedicine Evidence for Neurosurgery Follow-Up Care}

Thus far there has been little neurosurgery-specific evidence. Only two single-center studies have been published and they focused on telemedicine follow-up care of patients who have undergone elective neurosurgery. In terms of telemedicine, the results of these studies support its feasibility, safety, and effectiveness as well as patient acceptance, satisfaction, and convenience, largely related to decreased travel burden.

The most recent is a 2016 US pilot study in an urban setting (University of California, Los Angeles) of elective neurosurgery patients for selected procedures conducive to remote history and visual examination. ${ }^{8}$ The telemedicine intervention was a real-time videoconference conducted by an allied health professional for follow-up care during the first 90 days after patients underwent surgery; the professional used a standardized template focused on key clinical questions to identify patients' complications, to perform a functional status evaluation, and to provide 
education regarding signs and symptoms warranting inperson emergency room or clinic visits. Videoconferences were completed with 47 patients from home using required equipment: mobile phone, tablet, or laptop computer with a video camera. The results demonstrated high patient acceptance and satisfaction and no difference in emergency room visits, readmission rates at 30 and 90 days, and common symptoms reported when telemedicine patients were compared to 42 patients with in-person postoperative clinic follow-up visits.

The other study reported on the use of telemedicine for postoperative routine follow-up and semi-emergent conditions of neurosurgery patients from a tertiary care medical center in Bangalore, India. ${ }^{2}$ The majority of the study population lives below the poverty line and has to travel long distances to the center's outpatient clinic. The telemedicine specialist center in Bangalore provides realtime postoperative videoconferencing services for mostly routine follow-up care $(58 \%$ cranial and $42 \%$ spinal pathologies) to two nodal centers located in the Indian cities of Barrackpore, West Bengal, and Bhubaneshwar, Odisha. This descriptive study retrospectively analyzed clinical data from more than 1500 patients with over 3000 teleconsultations during a 6-year period beginning in 2007 . Based on their results, the authors concluded that videoconferencing for neurosurgical procedure follow-up care was efficient, safe, and cost-effective in an environment with significant resource constraints.

\section{New Neurosurgery Telemedicine Follow-Up Care Cost-Effectiveness Study}

A new study by Thakar and colleagues ${ }^{11}$ in the present issue is an important contribution as the first cost-effectiveness analysis of a telemedicine intervention in the followup care of patients who have undergone a neurosurgical procedure. Their study applied a novel decision-analytic and effectiveness measurement strategy retrospectively to 1200 consecutive patients from West Bengal, India, who underwent elective cranial and spinal neurosurgery during a 52-month period beginning January 1, 2013. This study used the same Bangalore, India, specialty center and telemedicine nodal center in Barrackpore, West Bengal, as the previously referenced study. Patients had their first postoperative in-person follow-up visit within 2 weeks of their surgery, and they were then advised to have their next follow-up visit at the telemedicine nodal center between 3 and 12 months postsurgery. The follow-up teleconsultation intervention was a targeted neurological examination conducted by a trained local physician, followed by a Skype teleconference, which included a high-resolution image transmission, with a neurosurgeon located at the specialty center.

Thakar and colleagues' decision analysis model compares two follow-up postoperative outpatient consultation options: real-time interactive teleconsultation from the nodal center and routine in-person care at the more distant outpatient specialty center that required 2-day train travel and a hotel stay for the patient and an attendant. Patient outcomes in terms of the medical benefit value are assumed to be identical for a successful teleconsultation and in-person specialty center consultation since both rely on the same neurosurgeon consultants, preventing use of the conventional quality-adjusted life-year (QALY) utility measure as an effectiveness outcome. Instead, effectiveness was measured by a utility preference score using a value scale of 0 to 1 for ease of access, quality of care, and overall experience. This score was applied to 4 consultation scenarios: 1) "successful" teleconsultation at nodal center $(0.9)$; 2) patient chooses in-person consultation at specialty center $(0.8)$; 3) "unsuccessful" teleconsultation requiring specialty center visit for additional evaluation and/or treatment services (0.6); and 4) "unsuccessful" teleconsultation requiring a local visit for additional nonneurosurgical care (0.4). A societal perspective for the analysis included direct and indirect costs incurred by the health system and patients, including potential lost earnings and the cost of having an attendant accompany the patient for all visits and, when visiting the specialty center, their travel, meals, and hotel lodging.

The result was that not only was teleconsultation costeffective but it was also a cost-saving alternative (i.e., lower cost and higher effectiveness). Cost-effectiveness for teleconsultation versus in-person specialty care was measured by an incremental cost-effectiveness ratio (ICER); the difference in cost was divided by the difference in effectiveness at base case values. Teleconsultation had a substantially lower per episode cost $(42.7 \%$ of in-person specialty care) with $97 \%$ efficiency or successful visit rate (i.e., care needs met without another visit required) yielding a higher overall utility (effectiveness) of 0.89 compared to 0.80 for in-person specialty care. Sensitivity analysis indicated threshold values at which teleconsultation would no longer be more cost-effective than in-person specialty consultation. The threshold values for the two most influential variables were $44 \%$ for the successful visit rate and 0.34 for the teleconsultation utility value, which hardly seem plausible compared to the study's well-supported base case values of $97 \%$ and 0.89 , respectively. Because a cost-saving result is a rare finding, additional and more representative evidence is needed to consider it credible.

\section{Conclusions}

The initial evidence on telemedicine for routine neurosurgical follow-up care offers suggestive support that telemedicine can be feasible, safe, and clinically and cost-effective with patient acceptance, satisfaction, and a reduced travel burden. Telemedicine may offer a win-win strategy for the specialty center clinic access problem caused by centralization of specialty services in a small number of tertiary care hospitals in large urban centers covering vast geographic areas, known as regionalization. While offering many advantages in both developed ${ }^{7}$ and developing countries, regionalization imposes a costly travel burden on many patients. An ironic, unintended consequence of regionalization is that the more successful neurosurgery centers are at providing surgical services, the greater the demand and corresponding access gap for low-value nonacute care services. Telemedicine may mitigate this gap in both developed- and developing-country settings, with the greatest relative benefit to rural, developing-country, low-income populations who bear the greatest travel burden. At the other extreme, telemedicine may be well ac- 
cepted and cost-effective for urban, developed-country, high-income, tech-savvy populations who value enhanced convenience and the reduced travel burden. ${ }^{8}$

This new telemedicine cost-effectiveness study for post-neurosurgical follow-up care is one of a small handful of studies for specialty surgery, ${ }^{1,5,12}$ and considerably more studies are needed to overcome a dearth of evidence. Moving telemedicine into the mainstream requires shifting its evidence base from promising toward generalizable to address decision-maker and policymaker information needs. Although telemedicine interventions may be considered affordable based on neurosurgery clinics' current staffing and technologies, and they may offer potential for significant patient and health system cost savings, there may be risks, concerns, and other costs constituting barriers to implementation. ${ }^{6}$ Future research demonstrating value sufficient to encourage adoption should provide rigorous, real-world practice comparative evaluation that is representative of neurosurgery telemedicine and in-person care costs in developing- and developed-country environments. A paradigm shift toward telemedicine and away from in-person specialist visits for routine neurosurgery follow-up care will depend on stakeholders' confidence in the net benefit of this substitution, which could potentially include reducing (the opportunity cost of) neurosurgeons' time spent on low-value outpatient clinic visits by replacing it with higher-value services, including surgeries.

https://thejns.org/doi/abs/10.3171/2018.2.FOCUS1889

\section{References}

1. Armstrong KA, Semple JL, Coyte PC: Replacing ambulatory surgical follow-up visits with mobile app home monitoring: modeling cost-effective scenarios. J Med Internet Res 16:e213, 2014

2. Dadlani R, Mani S, Jai Ganesh AU, Mohan D, Rajgopalan N, Thakar S, et al: The impact of telemedicine in the postoperative care of the neurosurgery patient in an outpatient clinic: a unique perspective of this valuable resource in the develop- ing world-an experience of more than 3000 teleconsultations. World Neurosurg 82:270-283, 2014

3. Gunter RL, Chouinard S, Fernandes-Taylor S, Wiseman JT, Clarkson S, Bennett K, et al: Current use of telemedicine for post-discharge surgical care: a systematic review. J Am Coll Surg 222:915-927, 2016

4. Hwa K, Wren SM: Telehealth follow-up in lieu of postoperative clinic visit for ambulatory surgery: results of a pilot program. JAMA Surg 148:823-827, 2013

5. Iqbal A, Raza A, Huang E, Goldstein L, Hughes SJ, Tan SA: Cost effectiveness of a novel attempt to reduce readmission after ileostomy creation. JSLS 21:e2016.00082, 2017

6. Kahn EN, La Marca F, Mazzola CA: Neurosurgery and telemedicine in the United States: assessment of the risks and opportunities. World Neurosurg 89:133-138, 2016

7. Ratcheson RA: Fast forwarding: the evolution of neurosurgery. The 2005 presidential address. J Neurosurg 103:585590,2005

8. Reider-Demer M, Raja P, Martin N, Schwinger M, Babayan D: Prospective and retrospective study of videoconference telemedicine follow-up after elective neurosurgery: results of a pilot program. Neurosurg Rev [epub ahead of print], 2017

9. Sanger PC, Hartzler A, Han SM, Armstrong CA, Stewart MR, Lordon RJ, et al: Patient perspectives on post-discharge surgical site infections: towards a patient-centered mobile health solution. PLoS One 9:e114016, 2014

10. Semple JL, Armstrong KA: Mobile applications for postoperative monitoring after discharge. CMAJ 189:E22-E24, 2017

11. Thakar S, Rajagopal N, Mani S, Shyam M, Aryan S, Rao AS, et al: Comparison of telemedicine with in-person care for follow-up after elective neurosurgery: results of a cost-effectiveness analysis of 1200 patients using patient-perceived utility scores. Neurosurg Focus 44(5):E17, 2018

12. Urquhart AC, Antoniotti NM, Berg RL: Telemedicine-an efficient and cost-effective approach in parathyroid surgery. Laryngoscope 121:1422-1425, 2011

\section{Disclosures}

The author reports no conflict of interest. 